\title{
Meson and Baryon Spectroscopy on the Lattice
}

\author{
D.G. Richards \\ Jefferson Laboratory, 12000 Jefferson Avenue Suite \#1, Newport News, VA 23606, USA
}

For the Hadron Spectrum Collaboration

\begin{abstract}
Recent progress at understanding the excited state spectrum of mesons and baryons is described. I begin by outlining the application of the variational method to compute the spectrum, and the program of anisotropic clover lattice generation designed for hadron spectroscopy. I present results for the excited meson spectrum, with continuum quantum numbers of the states clearly delineated. I conclude with recent results for the low lying baryon spectrum, and the prospects for future calculations.
\end{abstract}

Keywords: Spectroscopy, Lattice QCD

PACS: 12.38.Gc,11.15.Ga,14.20.Gk,14.40.Rt

In order to really understand QCD and hence test whether it is the complete theory of the strong interaction, we must know the spectrum of mesons and baryons that it implies and test those spectra against high quality data. The three valence quarks of baryons are emblematic of the non-abelian nature of QCD, and the determination of the $N^{*}$ spectrum is thus a key experimental effort. The observed spectrum of QCD provides little direct evidence of the presence of the gluons. However, QCD admits the possibility of exotic mesonic states of matter in which the gluonic degrees of freedom are explicitly exhibited. The search for such states will be an important component of the upgraded JLab@12GeV.

Given the intense experimental efforts in hadron spectroscopy, the need to predict and understand the hadron spectrum from first principles calculations in QCD is clear. Hence, an important goal of the lattice effort since its inception has been the study of the resonance spectrum of QCD, and more recently the calculation of the electromagnetic properties of low-lying resonances.

A comprehensive picture of resonances requires that we go beyond a knowledge of the ground state mass in each channel, and obtain the masses of the lowest states of a given quantum number. This we can accomplish through the use of the variational method $[1,2]$. Rather than measuring a single correlator $C(t)$, we determine a matrix of correlators

$$
C_{i j}(t)=\sum_{\vec{x}} \sum_{\vec{y}}\left\langle O_{i}(\vec{x}, t) O_{j}^{\dagger}(\vec{y}, 0)\right\rangle,
$$

where $\left\{O_{i} ; i=1, \ldots, N\right\}$ are a basis of interpolating operators with given quantum numbers. We then solve the generalized eigenvalue equation

$$
C(t) u=\lambda\left(t, t_{0}\right) C\left(t_{0}\right) u
$$


to obtain a set of real (ordered) eigenvalues $\lambda_{n}\left(t, t_{0}\right)$, where $\lambda_{0} \geq \lambda_{1} \geq \cdots \geq \lambda_{N-1}$. At large Euclidean times, these eigenvalues then delineate between the different masses

$$
\lambda_{n}\left(t, t_{0}\right) \longrightarrow e^{-M_{n}\left(t-t_{0}\right)}+O\left(e^{-\Delta M_{n}\left(t-t_{0}\right)}\right) .
$$

where $\Delta M_{n}=\min \left\{\left|M_{n}-M_{i}\right|: i \neq n\right\}$. The eigenvectors $u$ are orthogonal with metric $C\left(t_{0}\right)$, and a knowledge of the eigenvectors can yield information about the partonic structure of the states.

The (hyper-) cubic lattice does not possess the full rotational symmetry of the continuum. Thus in a lattice calculation, states at rest are classified not according to the spin $\left(J, J_{z}\right)$, but rather according to the irreducible representations (irreps) of the symmetry group of the cube; for states of higher spin, the different continuum degrees of freedom are distributed across several lattice irreps. Thus a crucial ingredient in our spectroscopy program has been the use of a large basis of interpolating operators, decomposed into their lattice irreps, enabling many energy levels to be reliably delineated. In the case of the meson spectrum below, we go further, and by using operators with definite spin in the continuum limit, are able to reliably identify the spins of the (single-hadron) states. Our ability to calculate correlation functions efficiently for such a large basis of operators has been greatly advanced through the development of a new method, "distillation"[3], for the construction of the interpolating operators.

The final ingredient in the spectroscopy effort has been the generation of so-called anisotropic lattices, with finer temporal than spatial discretisation, enabling the fall-off of the principle correlators introduced above to be discerned at short temporal separations.

\section{Anisotropic Clover Lattice Generation}

Precise calculations that can truly confront the experimental program require that we generate lattices with the correct number of light-quark flavors. The use of anisotropic lattices has proved essential in the reliable determination of the energy levels necessary to extract the resonance spectrum. Thus a crucial activity has been the generation of lattices with two flavors of fully dynamical light and a dynamical strange quark, using the clover fermion action, designed both for studies of the spectrum, and for the calculation of the scattering lengths important for understanding the nucleon-nucleon interaction. An important milestone was the tuning of the parameters of the action, beginning with the three-flavor theory[4].

A major challenge in calculations in lattice QCD is a procedure for specifying the values of the quark masses, and in particular those of the light $u / d$ and $s$ quarks, in a way that enables lattice calculations to be extrapolated to the physical values of these masses. This was accomplished through the introduction of a novel pair of dimensionless coordinates $l_{\Omega}$ and $s_{\Omega}$ that are primarily sensitive to the light and strange quark masses, 


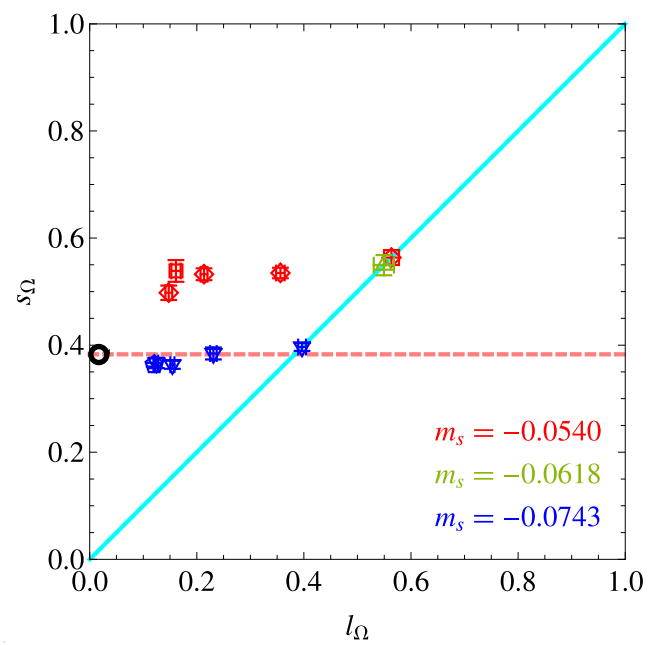

FIGURE 1. The figure shows the dynamical ensembles generated in [5] plotted in terms of the dimensionless coordinates $l_{\Omega}$ and $s_{\Omega}$, as described in the text. The solid line corresponds to the theory with three degenerate quark flavors, whilst the red horizontal line shows the approach to the physical quark masses; the black circle is the physical value of the coordinates.

respectively[5]:

$$
\begin{aligned}
l_{\Omega} & =\frac{9 m_{\pi}^{2}}{4 m_{\Omega}^{2}}, \\
s_{\Omega} & =\frac{9\left(2 m_{K}^{2}-m_{\pi}^{2}\right)}{4 m_{\Omega}^{2}} .
\end{aligned}
$$

The use of these coordinates is illustrated in Figure 1, showing the approach at fixed strange-quark mass to the physical values of the light $(u / d)$ quark masses.

Whilst the anisotropic lattices are designed to enable the investigation of the resonance spectrum of QCD, the low-lying spectrum, corresponding to the lightest states for each quantum number, provides an important benchmark for future calculations. The lefthand panel of Figure 2 shows a summary of the low-lying light-hadron masses compared with their experimental values; the right-hand panel shows the relative errors on the results compared to experiment[5]. It is this lattice-generation program that is at the core of our efforts to understand QCD spectroscopy.

\section{EXCITED MESON SPECTRUM}

The new Hall D of the JLab $12 \mathrm{GeV}$ upgrade centers on the study of meson states produced in photoproduction reactions in the GlueX experiment. Photoproduction has been proposed, within QCD-motivated models, as a favorable method for the production of exotic hybrid mesons, those mesons having $J^{P C}$ outside the set allowed to a fermionantifermion pair. The exotic hybrid hypothesis is that these quantum numbers arise from an excited gluonic field, in addition to a quark-antiquark pair. 

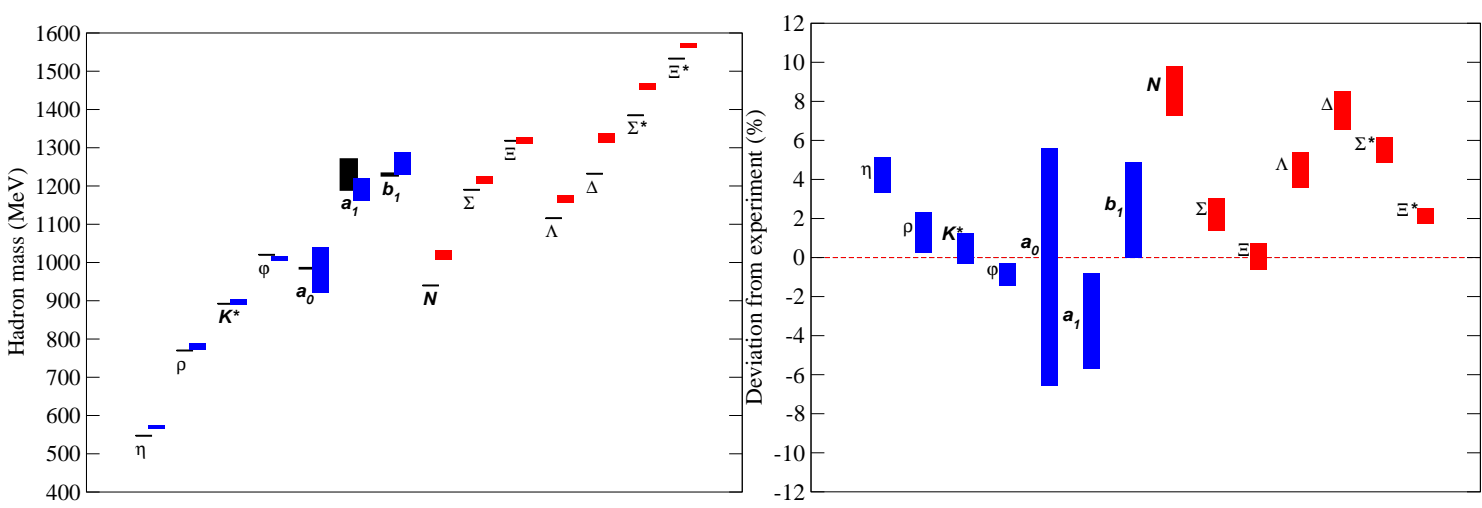

FIGURE 2. The left-hand panel shows a comparison between the calculated values of the low-lying hadrons and their physical values; the right-hand panel shows the percentage deviation from experiment.

A formidable barrier to the interpretation of lattice calculations of the spectrum has been the reduced cubic symmetry imposed by the lattice which makes the assignment of the continuum spins to the energy eigenstates problematic. The judicious choice of interpolating operators is crucial to the identification of the meson spins. Our starting point is a set of interpolating operators

$$
\mathscr{O}=\sum_{\vec{x}} \bar{\psi}(\vec{x}) \Gamma_{i} \overleftrightarrow{D}_{i} \overleftrightarrow{D}_{j} \ldots \psi(\vec{x})
$$

where $\overleftrightarrow{D}$ is a discretized covariant derivative, and $\psi(\bar{\psi})$ a quark (antiquark) field. From this set, we can construct a basis of interpolating operators $\mathscr{O}^{J, M}$ that in the continuum have definite spin,

$$
\left\langle 0\left|\mathscr{O}^{J M}\right| J^{\prime}, M^{\prime}\right\rangle=Z^{J} \delta_{J, J^{\prime}} \delta_{M, M^{\prime}}
$$

It is these operators that are now subduced to the lattice irreducible representations:

$$
\mathscr{O}_{\Lambda, \lambda}^{[J]}=\sum_{M} S_{\Lambda, \lambda}^{J, M} \mathscr{O}^{J, M}
$$

where $\Lambda, \lambda$ denote the lattice irrep. and its row respectively, and $S_{\Lambda, \lambda}^{J, M}$ are calculable coefficients. Whilst the resulting operators $\mathscr{O}_{\Lambda, \lambda}^{[J]}$ interpolate to any state whose spin is subduced to the irrep. $\Lambda$, we find that in practice the operator interpolates strongly to states of spin $J$, and that the resulting overlaps $Z^{J}$ are common across the different subductions; the method is described in detail in ref. [6]. Thus the operators $\mathscr{O}_{\Lambda, \lambda}^{[J]}$ retain a strong memory of their continuum antecedents, and this property enables us to identify the spins of the states for the effectively single-hadron states we have observed in the following calculation.

The availability of the large ensembles of $N_{f}=2+1$ anisotropic clover lattices, together with the efficient operator-construction admitted by "distillation", has enabled us to compute the isovector meson spectrum for states composed of the light and strange quarks[7, 6], at pion masses down to around $400 \mathrm{MeV}$. Masses of highly excited states 


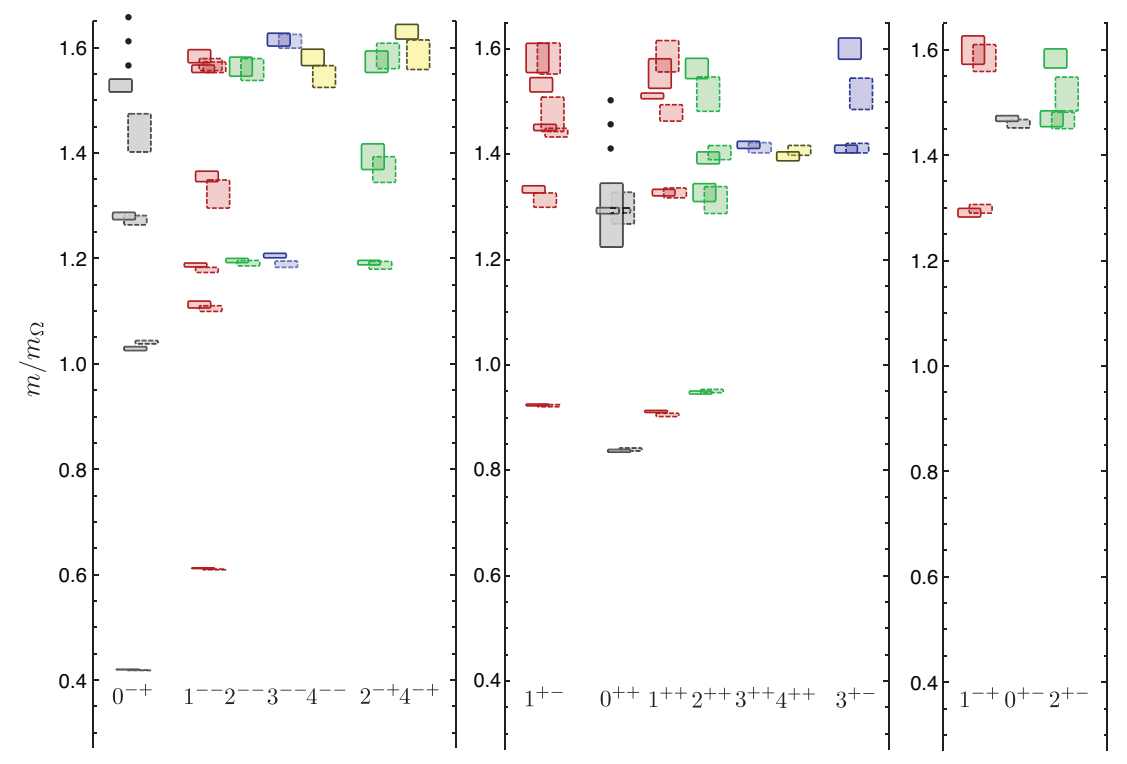

FIGURE 3. Spin-identified spectrum of isovector (octet) mesons in a calculation with three degenerate flavors of quarks of mass around that of the strange quark[6]. The calculation is performed at two spatial volumes: $16^{3}$ (solid) and $20^{3}$ (dashed) spectra agree well. Ellipses indicate that there are heavier states with a given $J^{P C}$ but that they are not well determined in this calculation. Note that the right-most panel corresponds to states of exotic quantum numbers.

have been calculated, with the quantum numbers reliably delineated, including for the first time in a lattice calculation states of spin 4, as illustrated in Figure 3 for the case of the three degenerate quark flavors. Furthermore, we see indications of hybrid states, but with conventional, non-exotic quantum numbers. Figure 4 summarizes the results on exotic states and compares those results with previous lattice QCD results from Refs. $[8,9,10,11,12,13]$.

The experimental implication of these results is important: there are likely mesons of exotic quantum numbers in a mass region accessible to the GlueX experiment. A notable feature of our calculation is that we see little evidence for the two-particle states that must be in the spectrum. In order to improve our sensitivity to such states, we believe it necessary to expand our basis of interpolating operators to include those with larger numbers of fermion fields, and this work is currently in progress.

\section{$N^{*}$ RESONANCES IN LATTICE QCD}

Baryons, containing three quarks, are emblematic of the non-Abelian nature of QCD, and of the three colors of the theory. An important goal in exploring baryons is attempting to discern the effective degrees of freedom that describe the spectrum; the search for so-called "missing resonances" focuses on whether the spectrum can be well described by a quark model, or whether an effective theory with fewer degrees of freedom, such as a quark-diquark picture, provides a more faithful description of the baryon spectrum.

The procedure for constructing interpolating operators for baryons was described in 


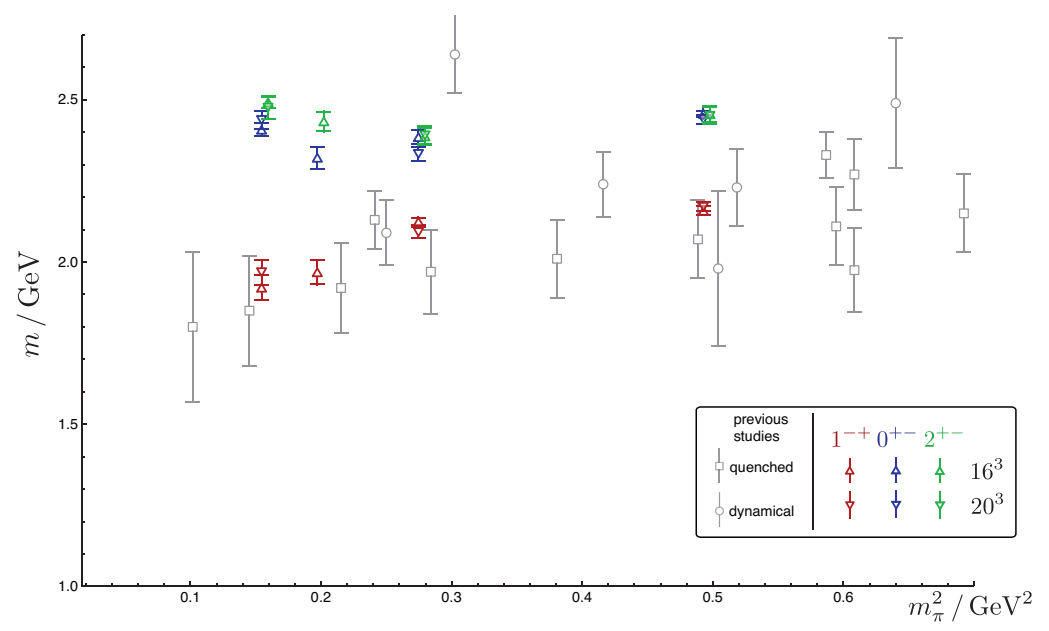

FIGURE 4. Summary of extracted isovector exotic states. For comparison $1^{-+}$results from Refs. $[8,9,10,11,12,13]$ are also plotted.

two papers, the first of which employed a Clebsch-Gordon approach[14], and the second an automated method allowing a very general basis of operators to be constructed [15]. There are three double-valued irreducible representations of the cubic group, denoted $G_{1 u / g}(2), H_{u / g}(4)$ and $G_{2 u / g}(2)$, where $g$ and $u$ refer to positive and negative parity, respectively, and the brackets contain the dimension of the irrep.; $G_{1}$ contains continuum spins $1 / 2,7 / 2, \ldots, H_{g}$ spins $3 / 2,5 / 2, \ldots$ and $G_{2}$ spins $5 / 2,7 / 2, \ldots$. Thus, at any fixed lattice spacing $a$, a state corresponding to spin-5/2 has four degrees of freedom in $H$, and two in $G_{2}$, with degeneracies between the energies in the two irreps. emerging in the continuum limit.

Our ability to extract the baryon resonances using the variational method was first shown in a calculation in the quenched approximation to QCD, using an anisotropic Wilson fermion action[16], and subsequently extended to a calculation using an anisotropic lattice with two mass-degenerate flavors of light quarks[17]. The "distillation" method once again allows an efficient means of computing the operators needed to apply the variational method.

The spectra of the Nucleon, $\Delta$ and $\Omega$ baryons on the $N_{f}=2 \oplus 1$ flavor lattices, have been calculated at three values of the light-quark masses corresponding to $m_{\pi}=$ 392,438 and $521 \mathrm{MeV}[18]$. The calculation of the nucleon and $\Omega$ spectra are shown in Figures 5 and 6, respectively. The operator basis is that obtained in reference [15] rather than one based on the subduction of continuum interpolating operators, and thus the calculated masses are shown according to their lattice irreps.. The pattern of states revealed is in general consistent with that observed in experiment; in the case of the $\Omega$, the experimental spin-parity assignment is only known for the lowest-lying state, and the determination of the spin-parity assignments is thus an important predictive opportunity for lattice QCD. The task of identifying the quantum numbers of the states will be greatly facilitated through the use of operators subduced from their continuum counterparts, in analogy with the meson calculations above[19]. Finally, we again note the need to include multi-hadron operators in our operator basis to resolve the multi-hadron states 
present in the spectrum.

\section{SUMMARY}

I have shown in this talk how the amalgam of anisotropic clover lattices, the use of the variational method, and the construction of a large basis of interpolating operators with known continuum behaviours is enabling the calculation of the excited spectrum of QCD, with the quantum numbers of the states identified. Future work will concentrate on the inclusion of multi-hadron states into the spectrum, the determination of the decay and scattering phase shifts, and the progression to the physical values of the light-quark masses.

\section{ACKNOWLEDGMENTS}

I am grateful to my colleagues in the Hadron Spectrum Collaboration, whose work is presented here. Supported by the U.S. DOE Contract No. DE-AC05-06OR23177 under which Jefferson Science Associates, LLC operates the Thomas Jefferson National Accelerator Facility. The Chroma software suite [20] was used to perform this work on clusters at Jefferson Laboratory and Fermi- lab using time awarded under the USQCD Initiative. The U.S. Government retains a non-exclusive, paid-up, irrevocable, worldwide license to publish or reproduce this manuscript for U.S. Government purposes.

\section{REFERENCES}

1. C. Michael, Nucl. Phys. B259, 58 (1985).

2. M. Luscher, and U. Wolff, Nucl. Phys. B339, 222-252 (1990).

3. M. Peardon, et al., Phys. Rev. D80, 054506 (2009), 0905.2160.

4. R. G. Edwards, B. Joo, and H.-W. Lin, Phys. Rev. D78, 054501 (2008), 0803.3960.

5. H.-W. Lin, et al., Phys. Rev. D79, 034502 (2009), 0810.3588.

6. J. J. Dudek, R. G. Edwards, M. J. Peardon, D. G. Richards, and C. E. Thomas, Phys. Rev. D82, 034508 (2010), 1004.4930.

7. J. J. Dudek, R. G. Edwards, M. J. Peardon, D. G. Richards, and C. E. Thomas, Phys. Rev. Lett. 103, 262001 (2009), 0909.0200.

8. C. Bernard, et al., Phys. Rev. D68, 074505 (2003), hep-lat/ 0301024.

9. C. W. Bernard, et al., Phys. Rev. D56, 7039-7051 (1997), hep-lat/9707008.

10. P. Lacock, and K. Schilling, Nucl. Phys. Proc. Suppl. 73, 261-263 (1999), hep-lat/9809022.

11. P. Lacock, C. Michael, P. Boyle, and P. Rowland, Phys. Lett. B401, 308-312 (1997), hep-lat/ 9611011.

12. C. McNeile, and C. Michael, Phys. Rev. D73, 074506 (2006), hep-lat/ 0603007.

13. J. N. Hedditch, et al., Phys. Rev. D72, 114507 (2005), hep-lat/ 0509106.

14. S. Basak, et al., Phys. Rev. D72, 074501 (2005), hep-lat/ 0508018.

15. S. Basak, et al., Phys. Rev. D72, 094506 (2005), hep-lat/ 0506029.

16. S. Basak, et al., Phys. Rev. D76, 074504 (2007), 0709.0008.

17. J. M. Bulava, et al., Phys. Rev. D79, 034505 (2009), 0901.0027.

18. J. Bulava, et al., Phys. Rev. D82, 014507 (2010), 1004.5072.

19. R. G. Edwards, et al. (2010), in preparation.

20. R. G. Edwards, and B. Joo, Nucl. Phys. Proc. Suppl. 140, 832 (2005), hep-lat/ 0409003. 

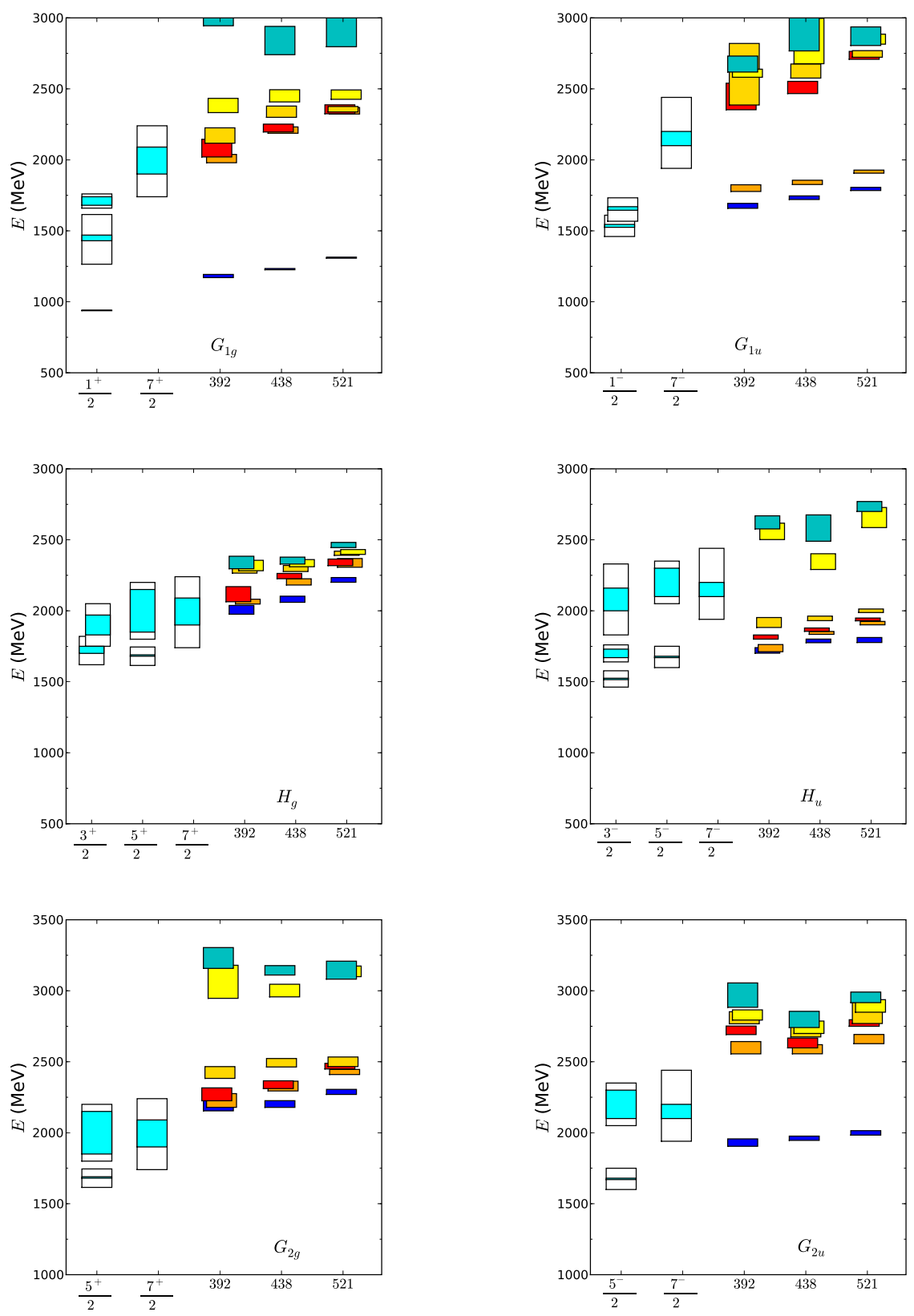

FIGURE 5. The nucleon $I=1 / 2$ spectrum at three values of the light-quark mass, corresponding to the $m_{\pi}=392,438$ and $521 \mathrm{MeV}$. The energies are shown according to the double-valued lattice irreps. $G_{1 g / u}, H_{g / u}$ and $G_{2 g / u}$, where $g$ and $u$ signify positive- and negative-parity, respectively. Also shown are the experimental spectra corresponding to each irrep, with the outer box representing the width, and the inner box the uncertainty on the Breit-Wigner mass. 

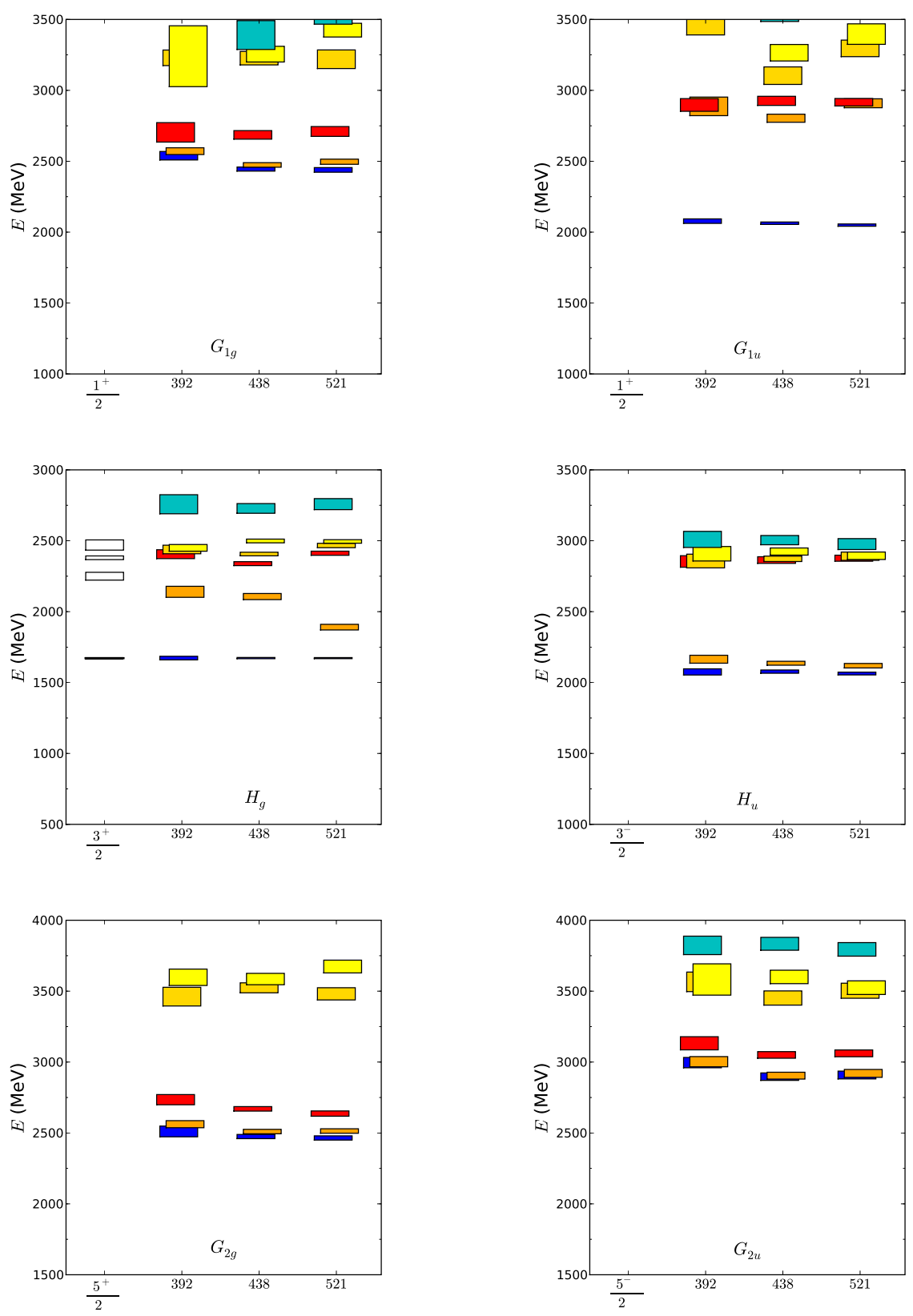

FIGURE 6. The Omega spectrum at three values of the light-quark mass, corresponding to the $m_{\pi}=$ 392,438 and $521 \mathrm{MeV}$. The energies are shown according to the double-valued lattice irreps. $G_{1 g / u}$, $H_{g / u}$ and $G_{2 g / u}$, where $g$ and $u$ signify positive- and negative-parity, respectively. Also shown are the experimental masses, with the height of the box representing the uncertainty on the Breit-Wigner mass; only for the ground state is the spin-parity assignment known. 